\title{
Comparação entre microdiluição e disco difusão para o teste de susceptibilidade aos antifúngicos contra Candida spp.
}

\section{Comparison of microdilution broth and disk diffusion for antifungal susceptibility testing against Candida spp.}

\author{
Antônio Alexandre de Vasconcelos Júnior ${ }^{1 *}$; Everardo Albuquerque Menezes²; \\ Francisco Afrânio Cunha3; Maria da Conceição dos Santos Oliveira Cunha; Bárbara \\ Helena Lima Braz; ${ }^{5}$ Ligya Guimarães Capelo ${ }^{6}$; Carlla Lorena Façanha Silva ${ }^{7}$
}

\section{Resumo}

A evolução da resistência fúngica pode ser avaliada pelo teste de susceptibilidade aos antifúngicos. Os protocolos mais utilizados para testes de susceptibilidade são a micro diluição em caldo (MDC), disco difusão (DD) e E-test. O objetivo principal deste estudo foi comparar os testes de sensibilidade realizados pelos métodos de MDC e DD para a anfotericina B e o fluconazol contra cepas de Candida spp. isoladas em Fortaleza/CE. Foram utilizadas 40 C. albicans, 50 C. tropicalis e 47 C. parapsilosis, isoladas de amostras de sangue e urina de pacientes atendidos no Hospital Geral de Fortaleza. Os testes foram realizados pelos métodos MDC e DD, de acordo com os protocolos M27-A3 e M44-A2 do CLSI. As metodologias foram comparadas e calculadas os percentuais de concordância. Para a anfotericina B e fluconazol, ocorreu um predomínio de cepas com susceptibilidade. O percentual de concordância para as metodologias foi acima de $97 \%$ e não foram detectados erros graves. A utilização de DD para realização de testes de susceptibilidade antifúngica pode ser aplicada na rotina laboratorial, pois é uma técnica fácil, barata, e de confiança quando comparada ao teste de MDC, sem comprometer os resultados para as cepas de C. albicans, C. tropicalis e C. parapsilosis.

Palavras-chave: Microdiluição. Disco difusão. Candida sp.

\footnotetext{
1 Laboratório de Microbiologia de Leveduras, Departamento de Análises Clínicas da Faculdade de Farmácia Odontologia e Enfermagem, Universidade Federal do Ceará, Brasil.*Contato principal para correspondência: alexandrevasconcelosjr@gmail. com

2 Laboratório de Microbiologia de Leveduras, Departamento de Análises Clínicas, Universidade Federal do Ceará, Brasil.

${ }^{3}$ Laboratório de Microbiologia de Leveduras, Departamento de Análises Clínicas, Universidade Federal do Ceará, Brasil.

${ }^{4}$ Laboratório de Microbiologia de Leveduras, Departamento de Análises Clínicas, Universidade Federal do Ceará, Brasil.

${ }^{5}$ Laboratório de Microbiologia de Leveduras, Departamento de Análises Clínicas, Universidade Federal do Ceará, Brasil.

${ }^{6}$ Laboratório de Microbiologia de Leveduras, Departamento de Análises Clínicas, Universidade Federal do Ceará, Brasil.

7 Curso de Química, Universidade Federal do Ceará, Brasil.
} 


\begin{abstract}
The evolution of fungal resistance can be evaluated by testing susceptibility to antifungal agents. The protocols for susceptibility testing most common are Broth Microdilution (BMD), Disk Diffusion (DD) and E-test. The objective of this study was to compare the susceptibility tests conducted by the BMD and DD methods for amphotericin B and fluconazole against Candida spp. isolated in Fortaleza/ CE. Were used 40 C. albicans, 50 C. tropicalis and 47 C. parapsilosis isolated from blood and urine samples of patients treated at Fortaleza General Hospital. The tests were conducted by the BMD and DD methods according to protocols M27-A3 and M44-A2 of the CLSI. These methods were compared and the percentages of agreement were calculated. For amphotericin B and fluconazole was a predominance of strains with reduced susceptibility. The percentage of agreement for the methods tested was above $97 \%$. No serious errors were detected. The use of DD for performing antifungal susceptibility testing can be applied in routine laboratory tests, since one technique is easy, inexpensive, and reliable when compared to the BMD without compromising the results for the strains of C. albicans, C. tropicalis and C. parapsilosis.
\end{abstract}

Keywords: Microdilution broth. Diffusion Disk. Candida sp.

\section{Introdução}

A epidemiologia das infecções fúngicas sistêmicas (IFS) está mudando, Candida albicans continua sendo a levedura mais importante (PATTERSON, 2005). No entanto, C. tropicalis, C. glabrata, C. parapsilosis e C. krusei têm sido cada vez mais isoladas e em alguns estudos ultrapassam os isolamentos de C. albicans (DIEKEMA et al., 2009; GOMES et al., 2010; HORN et al., 2009; MENEZES; MENDES; CUNHA, 2009; SELLAMI et al., 2011).

Os principais antifúngicos prescritos para o tratamento das IFS são o fluconazol e a anfotericina B (HORN et al., 2009). Candida spp. resistentes ao fluconazol têm sido detectadas em diversas partes do mundo (PFALLER, 2008, 2010b). No entanto, a resistência à anfotericina $\mathrm{B}$ não é comumente detectada, apesar dessa droga está em uso a mais de 50 anos (DIEKEMA et al., 2009; LANIADOLABORÍN; CABRALES-VARGAS, 2009).

Na última década foram implantados programas de vigilância da resistência fúngica, o mais importante deles é o ARTEMIS DISK, que monitora a resistência ao fluconazol e ao voriconazol em 134 instituições de 40 países, incluindo o Brasil, utilizando a metodologia de disco difusão (PFALLER et al., 2008, 2009a, 2009b, 2010b).
A evolução da resistência fúngica pode ser avaliada pelo teste de susceptibilidade aos antifúngicos. Há diversos protocolos para testes de susceptibilidade, no entanto os mais utilizados são a microdiluição em caldo (MDC), disco difusão (DD) e E-test (CAMPANA et al., 2011). Geralmente o MDC é um teste trabalhoso e que é menos utilizado, pois exige pessoal treinado, já o E-test é caro para ser aplicado à rotina laboratorial (CUENCAESTRELLA et al., 2011; PFALLER et al., 2010a). A alternativa é a técnica de DD, pois é simples, barata e reprodutível, portanto pode ser utilizada em laboratórios clínicos (GOMES et al., 2010; VASCONCELOS JÚNIOR; MENEZES; CUNHA, 2011).

O objetivo deste estudo foi comparar os testes de sensibilidade realizados pelos métodos de MDC e DD para a anfotericina B e o fluconazol contra cepas de Candida spp. isoladas em Fortaleza/CE.

\section{Material e Métodos}

\section{Leveduras utilizadas no estudo}

Foram utilizadas 137 Candida spp. (40 C. albicans, 50 C. tropicalis e 47 C. parapsilosis), isoladas amostras de sangue e urina de pacientes atendidos no Hospital Geral de Fortaleza, durante 
os anos de 2008 a 2010. As cepas estavam estocadas a $-20^{\circ} \mathrm{C}$ em meio Mueller-Hinton caldo com $50 \%$ de glicerina e pertencem à coleção do Laboratório de Microbiologia de Leveduras da Universidade Federal do Ceará. Para a realização dos testes, as Candida spp. foram descongeladas e semeadas duas vezes em ágar batata dextrose (Himédia-MumbaiÍndia) e em meio Chromoágar (Himédia-MumbaiÍndia), para garantir a pureza e a viabilidade das leveduras. A identificação das cepas foi realizada por testes de fermentação, assimilação de carboidratos, teste do tubo germinativo, micromorfologia em ágar arroz com tween 80. A identificação das cepas foi confirmada com a utilização do kit comercial ID 32C (bioMerieux - Paris, França) (GOMES et al., 2010; MENEZES; MENDES; CUNHA, 2009; VASCONCELOS JÚNIOR; MENEZES; CUNHA, 2011).

\section{Teste de Sensibilidade a Antifúngicos}

A susceptibilidade aos antifúngicos anfotericina B (Inlab-SP) e fluconazol(Sigma-EUA) foi testada por dois métodos diferentes, micro diluição em caldo RPMI(Cultilab-SP) (MDC) e disco difusão (DD). Para o teste de micro diluição em caldo, foi realizado o que recomenda o protocolo do CLSI M27-A3, foi utilizado RPMI caldo tamponado com ácido morfolino polissulfônico (MOPS) (Amresco-EUA). A anfotericina B foi solubilizada em dimetilsulfóxido (DMSO) e o fluconazol em água. Foram realizados testes com DMSO como controle para garantir que não houve interferência do diluente. A faixa testada para a anfotericina B variou de 16 a $0,03 \mu \mathrm{g} / \mathrm{mL}$ e o fluconazol de 64 a $0,25 \mu \mathrm{g} / \mathrm{mL}$. As placas foram inoculadas com as cepas e incubadas a $35^{\circ} \mathrm{C}$ por $24 \mathrm{~h}$, a leitura foi visual. A concentração inibitória mínima (CIM) da anfotericina $\mathrm{B}$ foi a menor concentração que não ocorreu crescimento fúngico, já para o fluconazol foi observado à concentração de antifúngico que causou uma redução de $50 \%$ do crescimento quando comparados com o controle (CLINICAL
AND LABORATORY STANDARDS INSTITUTE, 2008b, 2008c).

O teste de disco difusão foi realizado em meio ágar Muller-Hinton, com $2 \%$ de dextrose e 0,5 $\mu \mathrm{g} / \mathrm{mL}$ de azul de metileno, como recomendado pelo protocolo do CLSI M44-A2. Os discos de antifúngicos foram produzidos utilizando discos de papel de filtro de $6 \mathrm{~mm}$ e continham anfotericina B $(100 \mu \mathrm{g})$ e fluconazol $(25 \mu \mathrm{g})$. As leveduras foram suspensas em salina e semeadas no meio de cultura e depois foram colocados os discos. As placas foram incubadas a $35^{\circ} \mathrm{C}$ por $24 \mathrm{~h}$, após esse período os halos foram medidos (CLINICAL AND LABORATORY STANDARDS INSTITUTE, 2008a). O experimento foi realizado em três repetições. O controle de qualidade dos testes foi realizado com as cepas C. parapsilosis ATCC 22019 e C. krusei ATCC 6258.

\section{Critérios Interpretativos das CIMs}

A interpretação das CIMs para a micro diluição em caldo foi realizada de acordo com o protocolo M27-S3, cepa susceptível (S), CIM $\leq 8 \mu \mathrm{g} / \mathrm{mL}$ (fluconazol) e $<1 \mu \mathrm{g} / \mathrm{mL}$ (anfotericina B); susceptível dose dependente (SDD), CIM 16 a $32 \mu \mathrm{g} / \mathrm{mL}$ (fluconazol); resistente (R), CIM $\geq 64$ $\mu \mathrm{g} / \mathrm{mL}$ (fluconazol) e $>1,0 \mu \mathrm{g} / \mathrm{mL}$ (anfotericina B) (COSTA-DE-OLIVEIRA et al., 2008). Os critérios para a interpretação dos halos obtidos nos testes de disco difusão foram baseados no protocolo M44-A2: S, halo $\geq 19 \mathrm{~mm}$ (fluconazol) e $>10 \mathrm{~mm}$ (anfotericina B); SDD, halo de 15 a 18 $\mathrm{mm}$ (fluconazol); e R, halo $\leq 14 \mathrm{~mm}$ (fluconazol) e $<10 \mathrm{~mm}$ (anfotericina B) (CLINICAL AND LABORATORY STANDARDS INSTITUTE, 2008a, 2008b, 2008c; VASCONCELOS JÚNIOR; MENEZES; CUNHA, 2011).

\section{Análise dos resultados}

A comparação das duas metodologias foi realizada pela utilização da análise do erro, como recomendado pelo CLSI. A definição de erro usada 
neste estudo foi a seguinte: o Very Major Error (VME) ocorreu quando foi detectada uma cepa (R)-resistente pelo método da MDC (considerado padrão) e essa mesma cepa apresentou-se como (S) - sensível quando testada pelo método DD; o Major Error (ME), foi observado quando uma cepa se mostrou (S) por MDC e (R) por DD; e o Minor error (M) ocorre quando o resultado da MDC foi (SDD) e da DD foi (S) ou (R). Em geral, para métodos apresentarem boa correlação o VME deve ser $<1,5 \%$ e a concordância entre as metodologias deverão ser $>90 \%$ (CLINICAL AND LABORATORY STANDARDS INSTITUTE, 2008b).

\section{Resultados}

A principal levedura isolada de infecções fúngicas continua sendo C. albicans (PATTERSON, 2005). Dentre as cepas testadas de C. albicans, não foram detectadas cepas com CIM $>1,0 \mu \mathrm{g} /$
$\mathrm{mL}$ para a anfotericina $\mathrm{B}$, cepas com $\mathrm{CIM}>1,0$ para Anfotericina B são consideradas resistentes (COSTA-DE-OLIVEIRA et al., 2008). No entanto, uma cepa de C. albicans apresentou resistência a esse antifúngico pela metodologia de DD.

Outras leveduras muito importantes e frequentemente isoladas são C. tropicalis e C. parapsilosis (PFALLER et al., 2008). Para as cepas de C. parapsilosis, a resistência a anfotericina $\mathrm{B}$ foi detectada pelo método DD mais não foi confirmada pelo MDC. Quanto ao fluconazol, uma cepa foi categorizada com resistente pelo DD, mas esse fenótipo não foi confirmado pela MDC considerado padrão para o teste de susceptibilidade aos antifúngicos.

Quando se avalia os perfis de susceptibilidade das cepas isoladas no Ceará, de uma maneira global, observa-se que a resistência ainda é incomum no estado, como pode ser evidenciado na Tabela 1.

Tabela 1 - Correlação da susceptibilidade entre Micro diluição $\left(\mathrm{CIM}_{50 \%}\right)$ e Disco Difusão da Anfotericina B e do Fluconazol contra cepas de Candida spp.

\begin{tabular}{lcccc}
\hline \multicolumn{1}{c}{ Cepas (n) } & \multicolumn{2}{c}{ CIM $(\mu \mathrm{g} / \mathrm{ml})^{\mathrm{a}}$} \\
\hline & Anfotericina B & $\mathrm{DD}^{\mathrm{c}}(\mathrm{mm})$ & $\mathrm{MDC}$ & $\mathrm{DD}$ \\
\hline $\mathrm{MDC}^{\mathrm{b}}$ & $\mathrm{MG}^{\mathrm{d}}$ & $\mathrm{MG}$ & $\mathrm{MG}$ & $\mathrm{MG}$ \\
C. albicans (40) & 0,10 & 21 & 0,40 & 34 \\
C. tropicalis (50) & 0,10 & 20 & 0,50 & 30 \\
C. parapsilosis (47) & 0,10 & 18 & 0,25 & 27 \\
\hline
\end{tabular}

${ }^{a}$ Categorização dos perfil de susceptibilidade das cepas: Anfotericina B: (S), MIC $<1 \mu \mathrm{g} / \mathrm{mL}$ ( $>10 \mathrm{~mm}$ ); (R), MIC $>1$ $\mu \mathrm{g} / \mathrm{mL}(<10 \mathrm{~mm})$. Fluconazol: S, CIM $\leq 8 \mu \mathrm{g} / \mathrm{mL}(\geq 19 \mathrm{~mm})$; SDD, CIM 16 a $32 \mu \mathrm{g} / \mathrm{mL}(15-18 \mathrm{~mm}) ; \mathrm{R}, \mathrm{CIM} \geq 64 \mu \mathrm{g} /$ $\mathrm{mL}(\leq 14 \mathrm{~mm})$. Categorização entre os métodos: ${ }^{b} \mathrm{MDC}$ - Micro diluição em caldo RPMI realizado de acordo protocolo M27-A3. 'DD- Teste de Disco Difusão realizado de acordo com o protocolo M44-A2. ${ }^{\mathrm{M}}$ MG- Média Geométrica.

Fonte: Autores.

Para a anfotericina $\mathrm{B}$, ocorreu um predomínio de cepas com $\mathrm{CIM}<0,1 \mu \mathrm{g} / \mathrm{mL}$ e halos superiores a $18 \mathrm{~mm}$, que caracteriza a sensibilidade a esse importante antifúngico. Quanto ao fluconazol, a CIM ficou em torno de 0,25 e $0,50 \mu \mathrm{g} / \mathrm{mL}$ para as cepas testadas e os halos acima de $19 \mathrm{~mm}$, caracterizando 
sensibilidade para a maioria das cepas testadas.

Para se avaliar metodologias diferentes, mas que forneçam resultados semelhantes, existe diferentes técnicas e modelos matemáticos, dentre elas a mais recomendada é a categorização do erro, ou seja, o cálculo do percentual de discrepâncias e o percentual de concordância. Quando as metodologias apresentam \%concordância $>90 \%$ elas são equivalentes (CLINICAL AND LABORATORY STANDARDS INSTITUTE, 2008c). Todos esses dados foram calculados e encontram-se dispostos na Tabela 2.

Tabela 2 - Avaliação das Discrepâncias entre as metodologias de Micro diluição e Disco Difusão para a Anfotericina B e Fluconazol contra cepas de Candida spp.

\begin{tabular}{|c|c|c|c|c|c|c|c|c|c|}
\hline \multirow{3}{*}{$\underline{\text { Cepas (n) }}$} & \multirow{2}{*}{\multicolumn{2}{|c|}{ Antifúngicos }} & \multicolumn{3}{|c|}{ avaliados $^{\mathrm{a}}$} & \multicolumn{4}{|c|}{ \% Discrepâncias } \\
\hline & & & \multicolumn{3}{|c|}{ Fluconazol } & \multicolumn{4}{|c|}{ entre MDC e DD } \\
\hline & $\mathrm{S}$ & $\mathrm{R}$ & $\mathrm{S}$ & SDD & $\mathrm{R}$ & VME & $\mathrm{ME}$ & Minor & $\% \mathrm{C}^{\mathrm{c}}$ \\
\hline \multicolumn{10}{|c|}{ C. albicans (40) } \\
\hline $\mathrm{MDC}$ & 40 & 0 & 40 & 0 & 0 & & & & \\
\hline DD & 39 & 1 & 40 & 0 & 0 & $0 / 0$ & $2,5 / 0$ & $0 / 0$ & $97,5 / 100^{d}$ \\
\hline \multicolumn{10}{|c|}{ C. tropicalis (50) } \\
\hline MDC & 50 & 0 & 50 & 0 & 0 & & & & \\
\hline $\mathrm{DD}$ & 50 & 0 & 50 & 0 & 0 & $0 / 0$ & $0 / 0$ & $0 / 0$ & $100 / 100$ \\
\hline \multicolumn{10}{|c|}{ C. parapsilosis (47) } \\
\hline MDC & 47 & 0 & 47 & 0 & 0 & & & & \\
\hline $\mathrm{DD}$ & 46 & 1 & 44 & 2 & 1 & $0 / 0$ & $0 / 2,1$ & $0 / 0$ & $97,9 / 97,9$ \\
\hline
\end{tabular}

aPerfil de Susceptibilidade: Sensível (S); Sensível Dose-Dependente (SDD); Resistente (R). ${ }^{\text {CCategorização entre os }}$ métodos: VME, very major error; $\mathrm{ME}$, major error; $\mathrm{M}$, minor error.; ${ }^{\mathrm{c}} \mathrm{C} \mathrm{C}$ percentual de concordância entre as duas metodologias. ${ }^{\text {\% } \% ~ d e ~ c o n c o r d a ̂ n c i a ~ p a r a ~ a n f o t e r i c i n a ~ B / f l u c o n a z o l . ~ M D C-~ m i c r o d i l u i c ̧ a ̃ o ~ e m ~ c a l d o ~ e ~ D D-~ D i s c o ~ d i f u s a ̃ o ~}$ em ágar.

Fonte: Autores.

\section{Discussão}

Um estudo recente com 8.059 C. albicans, $99,2 \%$ das cepas apresentaram CIM para fluconazol, (PFALLER et al., 2010a), dado semelhante aos nossos resultados. Em um trabalho realizado no Brasil com 400 C. albicans isoladas de sangue, todas se mostraram susceptíveis ao fluconazol e a anfotericina B (MATTA et al., 2007).

Matta et al. (2007), avaliando C. parapsilosis e C. tropicalis no Brasil, não encontrou resistência ao fluconazol nem a anfotericina B. As cepas de C. tropicalis apresentaram susceptibilidade a anfotericina B e ao fluconazol. C. tropicalis costumam apresentar o fenômeno de trailling, que se caracteriza pelo crescimento em concentrações mais elevadas de antifúngicos do que aquelas determinadas pela CIM. No nosso estudo não detectamos trailling. Pela técnica de micro diluição em caldo, não detectamos resistência a esses antifúngicos. 
No entanto, em um recente trabalho realizado por Dalazen et al. (2011), ficou demostrado que em Santa Catarina ocorreu um alto índice de resistência para o fluconazol e anfotericina $B$, entre cepas orais e vaginais, isso pode ser explicado porque o estudo em questão envolvia cepas de C. krusei e C. glabrata que apresentam resistência natural ao fluconazol. Essas cepas não foram utilizadas no presente estudo, pois não possuímos isolados dessas espécies.

Em um estudo com 3.546 cepas de C. albicans, C. tropicalis e C. parapsilosis avaliadas pelo método do disco difusão, realizado no Brasil, a susceptibilidade ao fluconazol foi maior que 95\% (AZEVEDO et al., 2011). Observamos resultados semelhantes, todas as cepas de C. albicans e C. tropicalis apresentaram susceptibilidade ao fluconazol pela mesma técnica utilizada (Tabela 1 e Tabela 2). Percebe-se que as CIMs e os halos encontrados ainda estão dentro dos valores recomendados pelo CLSI e a resistência ainda não é um problema preocupante no Ceará. Entretanto, as cepas de C. parapsilosis apresentaram CIMs maiores, mas ainda sensíveis, e que requer uma atenção especial, principalmente quando se trata da susceptibilidade ao fluconazol. Pfaller et al. (2008), destacaram que C. parapsilosis pode apresentar susceptibilidade diferenciada dependendo da localização geográfica (PFALLER et al., 2008).

No nosso trabalho, mesmo com um número limitado de cepas, observamos que a concordância entre as metodologias foi maior que 97\% (Tabela 2). Pfaller et al., comparando as mesmas metodologias para o fluconazol contra 3.227 cepas de todas as partes do mundo, incluindo o Brasil, encontro um percentual de concordância por volta de $90 \%$ (PFALLER et al., 2009a).

Com base nos resultados da Tabela 2, observamos que o percentual de concordância para as metodologias testadas foi acima de $97 \%$, o que nos fornece a certeza de que a utilização de disco difusão para realização de testes de sensibilidade antifúngica, mesmo não fornecendo valores CIMs, pode ser uma alternativa importante frente ao teste de micro diluição em caldo, sem comprometer os resultados de susceptibilidade para as cepas de C. albicans, C. tropicalis e C. parapsilosis.

Metodologias que apresentam a concordância acima de $90 \%$ são consideradas ideais. Outro fator relevante é que no nosso estudo o very major error encontrado foi nulo, ficou abaixo de 1,5\% considerado ideal internacionalmente (CLINICAL AND LABORATORY STANDARDS INSTITUTE, 2008b).

Em outros estudos com Candida spp. isoladas no Brasil, foi encontrada uma concordância que se aproxima de $70 \%$ entre os métodos (DOTA et al., 2011; NEGRI et al., 2009), valor muito inferior ao que obtivemos. Todos esses estudos referemse ao fluconazol, pois a anfotericina B ainda se encontra em processo de padronização. O CLSI não definiu pontos de corte para esse antifúngico, os aqui sugeridos são somente para nortear o teste, muito ainda precisa ser feito com relação teste de sensibilidade a antifúngicos, sobretudo estudos envolvendo a anfotericina B.

Em conclusão, o nosso estudo mostrou, que a metodologia de disco difusão em ágar é relevante para ser aplicada na rotina laboratorial, pois é uma técnica fácil, barata, e de confiança quando comparada ao teste de micro diluição em caldo, pois mesmo não fornecendo valores de CIM precisamente pode ajudar no monitoramento da resistência fúngica, sendo de fundamental importância para uma terapia correta. Todavia, deve ser usada com cautela para outras cepas diferentes das citadas nesse estudo (KIRAZ et al., 2010), sendo necessários mais estudos que envolvam outras espécies.

\section{Agradecimentos}

Este trabalho foi financiado pelo Conselho Nacional de Desenvolvimento Científico e Tecnológico (CNPq). 


\section{Referências}

AZEVEDO, A. C.; BIZERRA, F. C.; DA MATTA, D. A.; DE ALMEIDA, L. P.; ROSAS, R.; COLOMBO, A. L. In vitro susceptibility of a large collection of candida strains against fluconazole and voriconazole by using the CLSI disk diffusion assay. Mycopathologia, Dordrecht, v. 171, n. 6, p. 411-416, 2011.

CAMPANA, E. H.; CARVALHAES, C. G.; BARBOSA, P. P.; MACHADO, A. M. O.; PAULA, A. M.; GALES, A. C. Avaliação das metodologias M.I.C.E. ${ }^{\circledR}$, Etest ${ }^{\circledR}$ e microdiluição em caldo para determinação da CIM em isolados clínicos. Jornal Brasileiro de Patologia e Medicina Laboratorial, Rio de Janeiro, v. 47, n. 2, p. 157164, 2011.

CLINICAL AND LABORATORY STANDARDS INSTITUTE. Method for antifungal disk diffusion susceptibility testing of yeasts: approved standard M44-A2. Wayne: Clinical and Laboratory Standards Institute, 2008a.

CLINICAL AND LABORATORY STANDARDS INSTITUTE. Reference method for broth dilution antifungal susceptibility testing of yeasts: approved standard M27-A3. 3. ed. Wayne: Clinical and Laboratory Standards Institute, 2008b.

CLINICAL AND LABORATORY STANDARDS INSTITUTE. Reference method for broth dilution Antifungal Susceptibility testing of yeasts; 3rd Informational Supplement, M27-S3. Wayne: Clinical and Laboratory Standards Institute, 2008c.

COSTA-DE-OLIVEIRA, S.; PINA-VAZ, C.; MENDONÇA, D.; GONÇALVES RODRIGUES, A. First portuguese epidemiological survey of fungaemia in a university hospital. European Journal of Clinical Microbiology \& Infectious Diseases, Berlin, v. 27, n. 5, p. 365-374, 2008.

CUENCA-ESTRELLA, M.; BASSETTI, M.; LASSFLORL, C.; RACIL, Z.; RICHARDSON, M.; ROGERS T. R. Detection and investigation of invasive mould disease. Journal of Antimicrobial Chemotherapy, London, v. 66, s. 1, p. i15-i24, 2011.

DALAZEN, D.; ZANROSSO, D.; WANDERLEY, L.; SILVA, N. L.; FUENTEFRIA, A. M. Comparação do perfil de suscetibilidade entre isolados clínicos de Candida spp. orais e vulvovaginais no Sul do Brasil. Jornal Brasileiro de Patologia e Medicina Laboratorial, Rio de Janeiro, v. 47, n. 1, p. 33-38, 2011.

DIEKEMA, D. J.; MESER, S. A.; BOYKEN, L. B.; HOLLIS, R. J.; KROEGER, J.; TENDOLKAR, S.; PFALLER, M. A. In vitro activity of seven systemically active antifungal agents against a large global collection of rare candida species as determined by clsi broth microdilution methods. Journal of Clinical Microbiology, Washington, v. 47, n. 10, p. 3170-3177, 2009.

DOTA, K. F. D.; FREITAS, A. R.; CONSOLARO, M. L.; SVIDZINSKI, T. I. E. Challenge for clinical laboratories: detection of antifungal resistance in Candida species causing Vulvovaginal Candidiasis. Laboratory Medicine, Chicago, v. 42, n. 2, p. 87-93, 2011.

GOMES, C. L.; CAVAlCANTE, J. E.; CUNHA, F. A.; AMORIM, L. N.; MENEZES, E. A. Identificação e perfil de sensibilidade de Candida spp isoladas de urina de pacientes com Candidúria em Iguatu-Ceará. Rio de Janeiro, v. 42, n. 3, p. 223-225, 2010.

HORN, D. L.; NEOFYTOS, D.; ANAISSIE, E. J.; FISHMAN, J. A.; STEINBACH, W. J.; OLYAEI, A. J.; MARR, K. A.; PFALLER, M. A.; CHANG, C. H.; WEBSTER, K. M. Epidemiology and outcomes of candidemia in 2019 patients: data from the prospective antifungal therapy alliance registry. Clinical Infectious Diseases, Chicago, v. 48, p. 1695-1703, 2009.

KIRAZ, N.; DAG, I.; OZ, Y.; YAMAC, M.; KIREMITCI, A.; KASIFOGLU, N. Correlation between broth microdilution and disk diffusion methods for antifungal susceptibility testing of caspofungin, voriconazole, amphotericin B, itraconazole and fluconazole against Candida glabrata. Journal of Microbiological Methods, Amsterdam, v. 82, n. 2, p. 136-140, 2010.

LANIADO-LABORÍN, R.; CABRALES-VARGAS, M. N. Amphotericin B: side effects and toxicity. Revista Iberoamericana de Micologia, Barcelona, v. 26, n. 4, p. 223-227, 2009.

MATTA, D. A.; DE ALMEIDA, L. P.; MACHADO, A. M.; AZEVEDO, A. C.; KUSANO, E. J.; TRAVASSOS, N. F.; SALOMÃO, R.; COLOMBO, A. L. Antifungal susceptibility of 1000 Candida bloodstream isolates to 5 antifungal drugs: results of a multicenter study conducted in São Paulo, Brazil, 1995-2003. Diagnostic Microbiology and Infectious Disease, New York, v. 57, n. 4, p. 399-404, 2007.

MENEZES, E. A.; MENDES, L. G.; CUNHA, F. A. Resistência a antifúngicos de Candida tropicalis isoladas no Estado do Ceará. Revista da Sociedade Brasileira de Medicina Tropical, Rio de Janeiro, v. 2, n. 3, p. 354-355, 2009.

NEGRI, M.; HENRIQUES, M.; SVIDZINSKI, T. I.; PAULA, C. R.; OLIVEIRA, R. Correlation Between E-tests, disk diffusion, and microdilution methods for antifungal susceptibility testing of candida species from infection and colonization. Journal of Clinical Laboratory Analysis, New York, v. 23, n. 5, p. 324-330, 2009. 
PATTERSON, T. F. Advances and challenges in the management of invasive mycoses. Lancet, London, v. 336, p. 1013-1025, 2005.

PFALLER, M. A. et al. Wild-type MIC distributions, epidemiological cutoff values and species-specific clinical breakpoints for fluconazole and Candida: time for harmonization of CLSI and EUCAST broth microdilution methods. Drug Resistance Updates,v. 13, p. 180-195, 2010a.

PFALLER, M. A.; DIEKEMA, D. J.; GIBBS, D. L.; NEWELL, V. A.; ELLIS, D.; TULLIO, V.; RODLOFF, A.; FU, W.; LING, T. A.; THE GLOBAL ANTIFUNGAL SURVEILLANCE GROUP. Results from the ARTEMIS DISK global antifungal surveillance study, 1997 to 2007 : a 10.5-Year Analysis of Susceptibilities of Candida Species to Fluconazole and Voriconazole as Determined by CLSI Standardized Disk Diffusion. Journal of Clinical Microbiology, Washington, v. 48. n. 4, p. 13661377, 2010b.

PFALLER, M. A.; DIEKEMA, D. J.; GIBBS, D. L.; NEWELL, V. A.; NG, K. P.; COLOMBO, A.; FINQUELIEVICH, J.; BARNES, R.; WADULA, J.; THE GLOBAL ANTIFUNGAL SURVEILLANCE GROUP. Geographic and temporal trends in isolation and antifungal susceptibility of candida parapsilosis: a global assessment from the artemis disk antifungal surveillance program, 2001 to 2005. Journal of Clinical Microbiology, Washington, v. 46. n. 3, p. 842-849, 2008.

PFALLER, M. A.; DIEKEMA, DJ.; GIBBS, DL.; NEWELL, VA.; BIJIE, H.; DZIERZANOWSKA, D.; KLIMKO, N. N.; LETSCHER-BRU, V.; LISALOVA, M.; MUEHLETHALER, K.; RENNISON, C.; ZAIDI, M.; THE GLOBAL ANTIFUNGAL SURVEILLANCE GROUP. Results from the ARTEMIS DISK global antifungal surveillance study, 1997 to 2007: 10.5-year analysis of susceptibilities of noncandidal yeast species to fluconazole and voriconazole determined by CLSI standardized disk diffusion testing. Journal of Clinical Microbiology, Washington, v. 47, n. 1, p. 117-123, 2009a.

PFALLER, M. A.; HAZEN, K. C.; MESSER, S. A.; BOYKEN, L.; TENDOLKAR, S.; HOLLIS, R. J.; DIEKEMA, D. J. Comparison of results of fluconazole and voriconazole disk diffusion testing for Candida spp. with results from a central reference laboratory in the ARTEMIS DISK Global Antifungal Surveillance Program. Diagnostic Microbiology and Infectious Disease, New York, v. 65, p. 27-34, 2009b.

SELLAMI, A.; SELLAMI, H.; NÉJI, S.; MAKNI, F.; ABBES, S.; CHEIKHROUHOU, F.; CHELlY, H.; BOUAZIZ, M.; HAMMAMI, B.; BEN JEMAA, M.; KHALED, S.; AYADI, A. Antifungal susceptibility of bloodstream Candida isolates in Sfax hospital: Tunisia. Mycopathologia, Dordrecht, v. 171, p. 417-422, 2011.

VASCONCELOS JÚNIOR, A. A.; CUNHA, F. A.; MENEZES, E. A. Chromogenic medium for direct susceptibility testing of Candida spp. Isolated from Urine. Mycopathologia, Dordrecht, v. 172. n. 2, p. 125130, 2011.

Recebido em 15 de novembro de 2011 Aceito em 25 de janeiro de 2012 